\title{
Visualisation et mesures expérimentales dans un jet rond évoluant à nombre de Reynolds modéré
}

\author{
Yassine Zaouali ${ }^{1, a}$, Jacques JaY $^{2}$ et Habib Ben Aissia ${ }^{1}$ \\ 1 Unité de Métrologie en Mécanique des Fluides et Thermique (03/UR/11-09), École Nationale d'Ingénieurs de Monastir, \\ Tunisie \\ 2 Centre de Thermique de Lyon (CETHIL - UMR CNRS 5008), Institut National des Sciences Appliquées de Lyon, France
}

Reçu le $1^{\text {er }}$ juin 2009 , accepté le 30 novembre 2009

\begin{abstract}
Résumé - L'objectif de ce travail est d'étudier les instabilités de Kelvin-Helmholtz apparaissant dans un jet de fluide monophasique évoluant à nombres de Reynolds modérés. L'étude porte sur la région proche de la buse d'éjection (la zone de transition), et elle est localisée sur l'évolution dans l'espace et dans le temps des instabilités aérodynamiques spécifiques à ce type d'écoulement. Dans cette étude expérimentale, les instabilités sont décrites qualitativement puis quantitativement à l'aide de la technique de tomographie par plan laser associée aux opérateurs de traitement d'images. On s'intéresse en particulier à l'étude des structures générées par l'instabilité des jets ainsi que la détermination de leurs paramètres caractéristiques.
\end{abstract}

Mots clés : Jet axisymétrique / nombre de Reynolds modéré / tomographie laser / instabilités de Kelvin-Helmholtz / traitement d'images

\begin{abstract}
Visualization and experimental measures in a round jet evolving at moderate Reynolds number. The objective of this work is to study the Kelvin-Helmholtz instabilities appearing in a monophase fluid jet evolving at moderate Reynolds numbers. The study concerns at the near region of the nozzle exit (the transition zone), and it's localized on the evolution in the space and in the time of aerodynamic instabilities specific to this type of out-flow. In this experimental survey, the instabilities are described qualitatively then quantitatively by technique of tomography by laser plan associated to operators of image processing. We are interested in particular in the study of the structures generated by the instability of the jets as well as the determination of their characteristic parameters.
\end{abstract}

Key words: Axisymmetric jet / moderate Reynolds number / laser tomography / Kelvin-Helmholtz instabilities / image processing

\section{Introduction}

Dans l'industrie, les écoulements de type jet rond évoluant à nombre de Reynolds modéré sont présents dans plusieurs applications telles que la pulvérisation, l'isolation thermique, la soudure dans les milieux aérodynamiques et hydrodynamiques, le séchage par jet rond unitaire ou en réseau matriciel, etc.

La plupart des travaux de recherche expérimentaux sur les jets traitent des nombres de Reynolds élevés et ainsi concernés par la turbulence. L'étude d'un jet laminaire n'est pas facile en raison de la sensibilité extrême de ces jets aux petites perturbations qui mènent aux instabilités et produisent finalement la turbulence (Batchelor [1],

\footnotetext{
a Auteur pour correspondance :

yes.zaouali@laposte.net
}

Crow [2], Cohen [3], Mollendorf [4], Ben Aissia [5], Fontaine [6]). Ces instabilités peuvent évoluer sous différents aspects selon la nature d'écoulement et les conditions expérimentales. La connaissance des critères de la transition laminaire-turbulent est importante aussi bien d'un point de vue fondamental que par les applications. Les frontières d'un jet dans la phase de transition sont instables, et une définition appropriée des paramètres régissant la transition à la turbulence n'est pas évidente.

L'étude de la vaste littérature consacrée aux instabilités du jet axisymétrique montre que les mécanismes de transition, la variation des modes instables selon le nombre de Reynolds, aussi bien que la détermination de l'étendue de la zone laminaire, demeurent encore des sujets ouverts. L'étude du comportement de l'écoulement dans cette zone est importante pour la compréhension du 


\section{Nomenclature}

\begin{tabular}{|lll|}
\hline$d$ & diamètre de la buse & $\mathrm{m}$ \\
$t$ & temps & $\mathrm{s}$ \\
$U$ & vitesse longitudinale & $\mathrm{m} \cdot \mathrm{s}^{-1}$ \\
$x$ & coordonnée longitudinale & $\mathrm{m}$ \\
$y$ & coordonnée transversale & $\mathrm{m}$ \\
$\alpha$ & angle d'amplification & $\circ$ \\
$\lambda$ & longueur d'onde & $\mathrm{m}$ \\
Indice & & \\
0 & sortie de la buse & \\
Nombre adimensionnel & & \\
$\operatorname{Re}$ & nombre de Reynolds & \\
\hline
\end{tabular}

jet entier. En effet, c'est dans la zone de transition que les instabilités se développent dans la couche de mélange et produisent des structures tourbillonnaires pas facilement prévisibles par la théorie pour des bas nombres de Reynolds. C'est une des raisons pour lesquelles la majorité des analyses théoriques traite le cas turbulent dans la zone de proche sortie du jet (Danaila [7]).

L'avantage d'une longue région laminaire est de mettre en évidence l'évolution des instabilités. Il est important d'obtenir des bonnes données expérimentales pour la validation des simulations récentes (Ben Aissia [5], Hinze [8], Ben Aissia [9], Ravier [10]) qui semblent donner des résultats au moins qualitativement corrects. Relativement, la littérature fait état de peu d'expériences dans ce cas (Crow [2], Becker [11]).

La majorité des études analytiques, numériques et expérimentales menées dans le cas d'un jet axisymétrique, considère que des nombres de Reynolds supérieurs à 300 (Mattigly [12]) sont suffisants pour que les effets non visqueux dominent les effets visqueux. Des instabilités primaires dans ce cas sont caractérisées par les ondes de Kelvin-Helmholtz. Autrement, dans la majorité des études expérimentales, la dynamique spatiale du jet est dominée dans une large gamme de nombres de Reynolds, par la présence des structures tourbillonnaires axisymétriques dans la zone de cisaillement, et qui sont convectées en aval.

À notre connaissance, dans le cas d'un jet laminaire axisymétrique libre évoluant naturellement, on ne dispose pas de visualisation du mécanisme de formation des instabilités de Kelvin-Helmholtz à partir de l'un ou l'autre des modes d'instabilité les plus amplifiés (sinueux ou variqueux), ni sur les tourbillons antisymétriques, ni sur les scénarios de leurs grossissements.

Les modes d'instabilité ont été observés dans quelques études expérimentales. Les premiers travaux de Reynolds [13] dans le cas d'un jet d'eau submergé dans un large réservoir montrent l'existence des ondulations sinusoïdales à une longueur d'onde élevée qui domine l'écoulement pour des nombres de Reynolds compris entre 150 et 300. Becker et Massaro [11] ont pu observer, pour $R e=1690$, une ondulation sinusoïdale à une distance de la sortie de la buse approximativement égale à 5 fois le diamètre. Crow et Champagne [2] décrivent d'une façon qualitative sans présenter de visualisations, le passage du mode sinueux aux modes intermédiaires ayant la forme du tire-bouchon puis du vilebrequin, et finalement au mode variqueux, quand le nombre de Reynolds change de 100 à 1000 .

Il découle de cette étude bibliographique que l'évolution de la forme du jet du mode sinueux au mode variqueux, dépend non seulement du nombre de Reynolds, mais également du dispositif expérimental et du milieu environnant le jet.

Nous proposons dans notre travail de mettre en œuvre des moyens expérimentaux adaptés à l'étude des instabilités dans la zone de transition d'un jet axisymétrique à faible nombre de Reynolds, et d'apporter des informations aussi bien qualitatives que quantitatives, sur la formation des instabilités de Kelvin-Helmholtz.

\section{Dispositif expérimental}

L'écoulement étudié est un jet d'air libre descendant à faible nombre de Reynolds et qui débouche d'une buse circulaire, de diamètre intérieur $d=12,4 \mathrm{~mm}$, dans une enceinte à air au repos.

Le dispositif expérimental de génération du jet est constitué essentiellement d'un compresseur alimentant un réservoir tampon, d'une vanne pointeau de contrôle du débit d'air, d'une série de détendeurs régulateurs de débit, et d'une chambre de tranquillisation formée d'un divergent, d'un nid d'abeille de section hexagonale de $6 \mathrm{~mm}$ de côté, de trois grilles de très faible diamètre et d'une buse d'injection. Cette dernière présente un convergent correctement profilé permettant d'obtenir un profil uniforme de la vitesse à la section d'éjection. L'ensemble du système générant le jet est localisé dans une cellule en plexiglas d'un volume d'environ $6 \mathrm{~m}^{3}$, et dont les parois intérieures ont été couvertes de plaques de liège et de carton ondulé dans le but d'isoler le jet de toutes perturbations externes (Fig. 1).

Ce jet est ensemencé par de la fumée issue de la combustion de bâtonnets d'encens. Les particules de la fumée d'encens ont des diamètres variant entre 0,5 et $5 \mu \mathrm{m}$ et sont de densité très proche de celle de l'air dans le but de suivre fidèlement l'écoulement.

Le système d'éclairage et de génération de la nappe laser est composé d'un laser à argon continu pouvant délivrer des puissances allant jusqu'à $7 \mathrm{~W}$ et d'un système optique monté en aval de la source laser permettant l'obtention d'une fine tranche laser de $1 \mathrm{~mm}$ d'épaisseur. Une caméra Pulnix TM-6703 (partial progressive scan) est placée perpendiculairement à la nappe éclairée. Elle permet d'enregistrer des séquences d'images pouvant atteindre une fréquence de 220 images par seconde pour une résolution de 640 par 100 pixels.

Un système d'Anémométrie Laser à effet Doppler (LDA) permet de mesurer la vitesse du jet à la sortie de la buse d'injection (Ben Aissia [14]). 


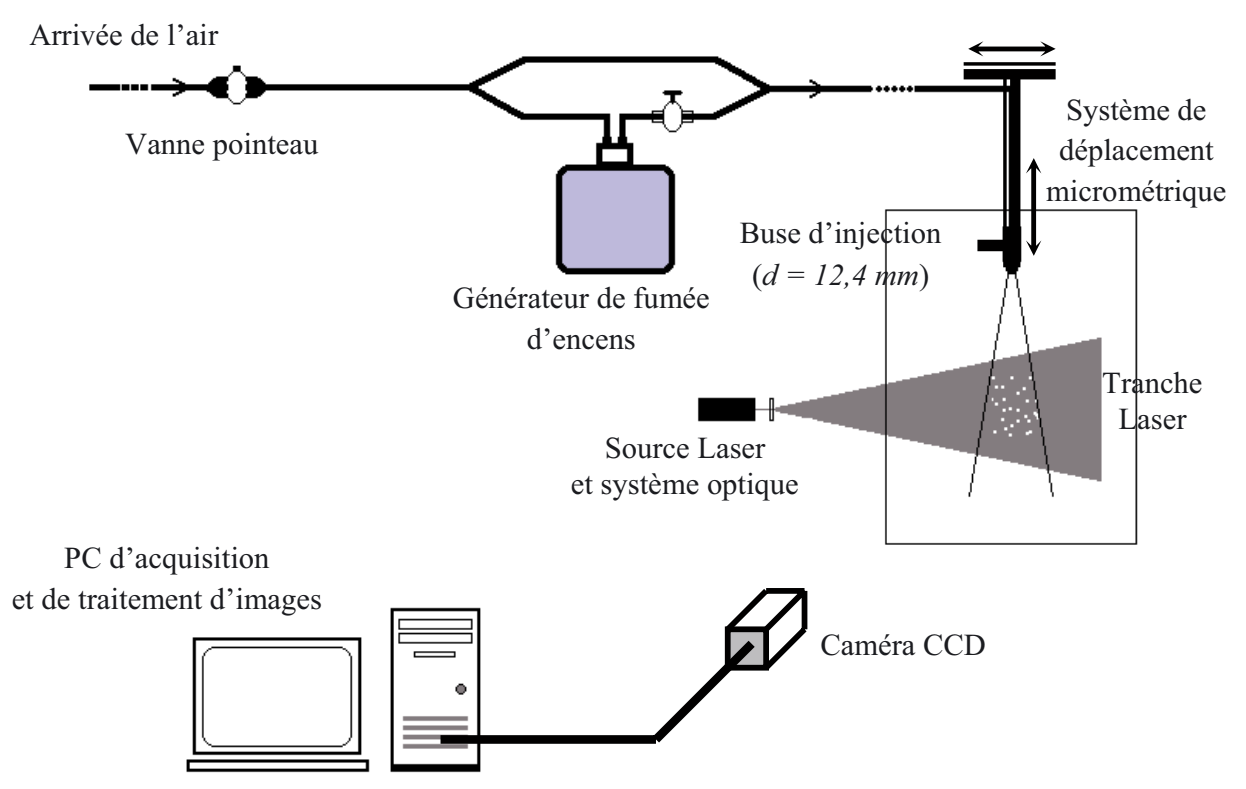

Fig. 1. Schéma de l'installation du jet d'air.

\section{Résultats et discussions}

\subsection{Visualisation du jet par tomographie laser}

Sur la figure 2, nous présentons quelques visualisations expérimentales du jet prises et enregistrées pour différents nombres de Reynolds (de 800 à 1800). Ces images montrent que le jet peut être divisé en trois zones : une zone laminaire, une zone de transition et une zone de turbulence ou de chaos. La partie laminaire a déjà été étudiée (Ben Aissia [14], Zaouali [15]) au moyen de la technique de PIV (vélocimétrie par image de particules). Dans le présent travail, nous allons nous intéresser à la zone intermédiaire qui sépare la partie laminaire de la partie turbulente.

Cette zone de transition est caractérisée par des instabilités pour lesquelles le mode le plus amplifié dépend du nombre de Reynolds basé sur le diamètre de la buse de sortie et la vitesse de l'injection. En effet, dans cette zone, la morphologie du jet visualisée pour un nombre de Reynolds de 830 est totalement différente de celle pour un Reynolds de 1800. Sur ces images, nous remarquons que les premières instabilités qui apparaissent sont le mode sinueux ou (et) le mode variqueux suivis par des instabilités de Kelvin-Helmholtz (K-H) antisymétriques, visibles pour des nombres de Reynolds faibles (Zaouali [15]), ou symétriques obtenues pour des nombres de Reynolds modérés.

\section{2 Évolution des instabilités de Kelvin-Helmholtz}

L'acquisition des images à une fréquence de $220 \mathrm{~Hz}$ nous a permis de suivre l'évolution temporelle des instabilités de Kelvin-Helmholtz symétriques. Nous présentons sur la figure 3, une séquence d'images successives acquises pour un nombre de Reynolds $R e=1700$.

Le suivi sur ces images d'une structure ou d'un « motif depuis son apparition (le motif à suivre est marqué par un tiré blanc sur la séquence d'images), montre que ce dernier prend naissance sous forme d'une crête dans la partie variqueuse du jet (à $x / d \approx 1,3$ pour $R e=1700$ ). Tout en se déplaçant dans le sens de l'écoulement, ce motif, en contact avec le fluide ambiant, est creusé par le creux juste en amont. Ceci crée un enroulement aboutissant à une structure tourbillonnaire qui commence alors à se former et s'agrandir au cours du temps. Plus en aval, la structure se détache du reste du jet et fusionne dans d'autres structures pour détruire l'axisymétrie de l'écoulement et passer au régime turbulent.

Il est à noter qu'à des fréquences d'acquisition plus faibles (120 images par seconde par exemple), les essais ont révélé des difficultés pour le suivi de l'évolution temporelle des structures dans le jet. Cependant, à une fréquence de 500 images par seconde, l'analyse des images nous montre les mêmes aspects de l'écoulement.

Nous remarquons aussi, dans certains cas, que deux motifs successifs peuvent se rattraper au cours de leur convection sur le bord du jet et se mélangent à la fin de la zone de transition pour créer le désordre et par suite le passage à la turbulence. La figure 4 illustre un exemple de séquence d'images sur lesquelles nous pouvons visualiser le parcours de deux motifs (marqués par les tirés blancs sur la figure) en fonction du temps.

Sur la figure 5, nous présentons une séquence d'images obtenue pour un nombre de Reynolds égal à 1300 et avec 


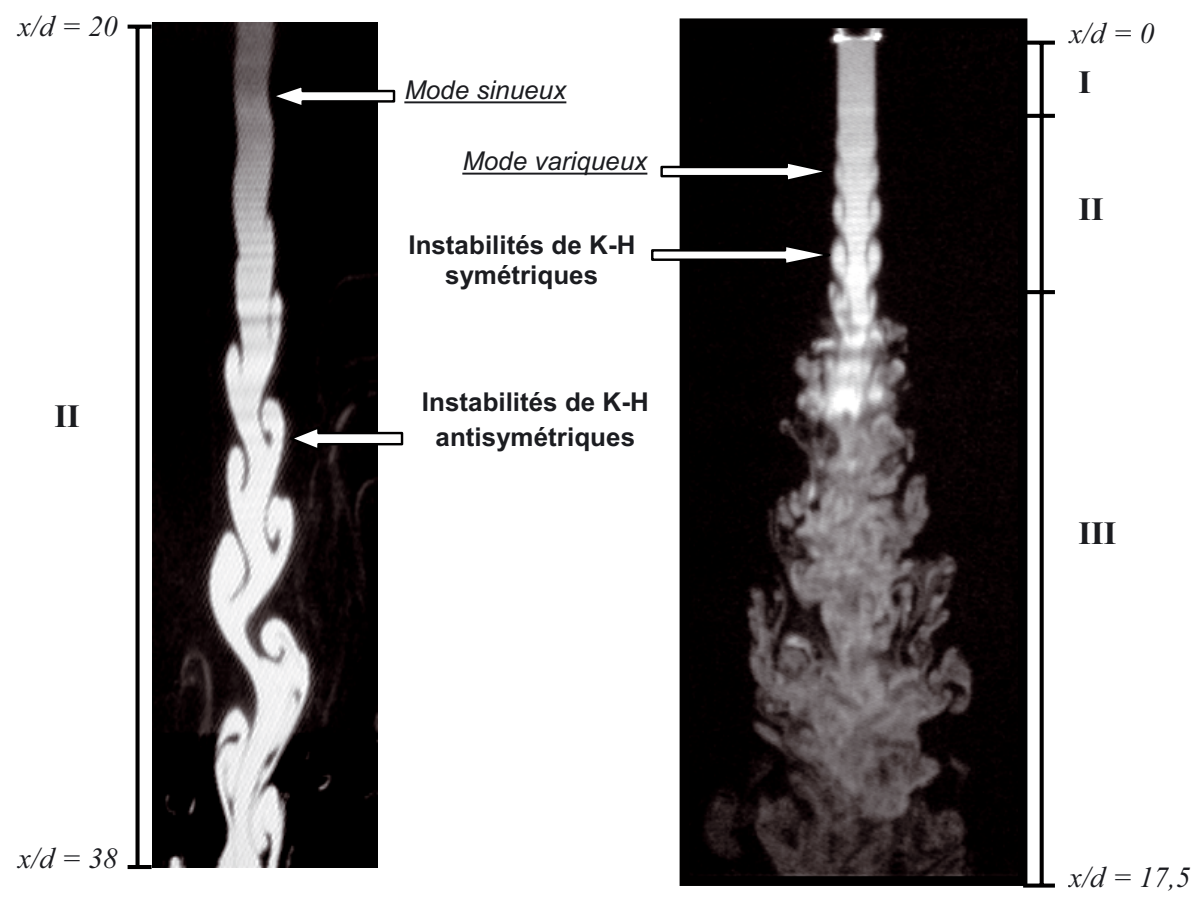

(a) $\operatorname{Re}=830$

(b) $\operatorname{Re}=1800$

I : Zone laminaire

II : Zone de transition

III : Zone de turbulence

Fig. 2. Visualisation expérimentale des zones caractéristiques du jet.

une fréquence d'acquisition de 220 images par seconde. Sur ces images, nous pouvons visualiser la formation d'un tourbillon à l'intérieur de la structure par enroulement sur lui-même. On note aussi le phénomène de grossissement de la structure d'une image à une autre tout en s'éloignant de la sortie de la buse. En effet, les structures tourbillonnaires augmentent de taille par induction réciproque de deux tourbillons consécutifs, au fur et à mesure qu'elles sont convectées vers l'aval. Cette augmentation de la taille ralentit le mouvement de la structure, tandis que la structure en amont est relativement accélérée. Le mouvement relatif qui s'en suit va vers la fusion des deux structures tourbillonnaires. Il s'agit ici du phénomène d'appariement vortical (Ho [16]), identiquement visible pour les autres nombres de Reynolds.

Il est à signaler que sur cette figure, la détection des frontières est réalisée par filtrage par gradient de Sobel sur l'image extraite (le motif à suivre est marqué par un tiré blanc sur la séquence d'images).

\subsection{Mesures des paramètres des instabilités de Kelvin-Helmholtz}

\subsubsection{Principe}

Le principe de mesure repose sur la localisation des points particuliers sur la ligne frontière du jet. Sur les images enregistrées, on peut distinguer quatre formes des étapes successives de l'évolution d'une même instabilité : « ondulation », « séparation naissante », « séparation avancée » et « rotation ». Sur chacune de ces formes, nous pouvons distinguer des points particuliers : les bosses $\ll$ B , les creux $« \mathrm{C} »$ et les pointes $\ll \mathrm{P} »($ Fig. 6$)$.

À partir de la localisation des points $\mathrm{B}_{1}, \mathrm{~B}_{1}^{\prime}$ et $\mathrm{B}_{1}^{\prime \prime}$ sur une même image, nous pourrons déterminer la longueur d'onde $\lambda$ des instabilités. Alors que sur deux images successives 1 et 2 décalées d'un délai de temps $\Delta t$, nous obtenons la vitesse de chacun de ces points par mesure des distances $\mathrm{B}_{1}-\mathrm{B}_{2}, \mathrm{~B}_{1}^{\prime}-\mathrm{B}_{2}^{\prime}, \mathrm{B}_{1}^{\prime \prime}-\mathrm{B}_{2}^{\prime \prime} \mathrm{C}_{1}-\mathrm{C}_{2}, \mathrm{C}_{1}^{\prime}-\mathrm{C}_{2}^{\prime}, \ldots$ Ceci nous donnera des informations sur la taille des tourbillons générées ainsi que leurs vitesses de convection.

\subsubsection{Ordre de grandeur par mesure directe}

Une mesure directe des distances entre les points B a été réalisée directement sur les images afin d'obtenir un ordre de grandeur (à 2 pixels près). Les résultats donnés dans le tableau 1 représentent des moyennes sur 200 images successives qui défilent à 220 images par seconde. Les valeurs sont données en relatif vis-à-vis du diamètre $d$ de la buse. Trois nombres de Reynolds sont choisis et la vitesse d'injection $U_{0}$ a été mesurée par LDA.

D'après ces résultats, la vitesse de phase des instabilités de Kelvin-Helmholtz est de l'ordre de $U \approx 0,65 U_{0}$. Quant à la longueur d'onde moyenne, elle est estimée à $\lambda \approx d$. La fréquence d'apparition de l'instabilité est alors de 0,65 , c'est-à-dire qu'un nouveau tourbillon est formé à une période spatiale adimensionnée de 1,5. 


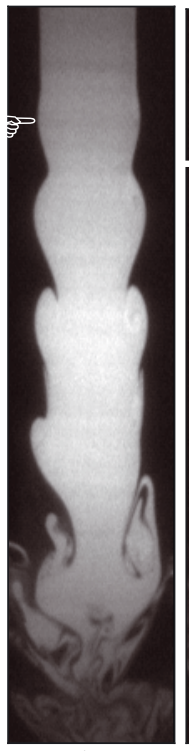

$-1-$

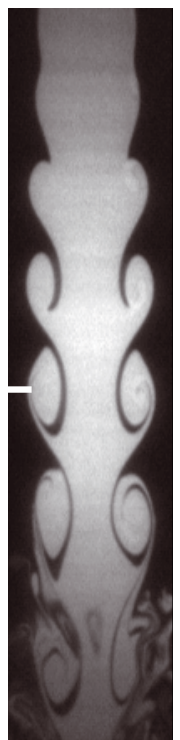

$-7-$

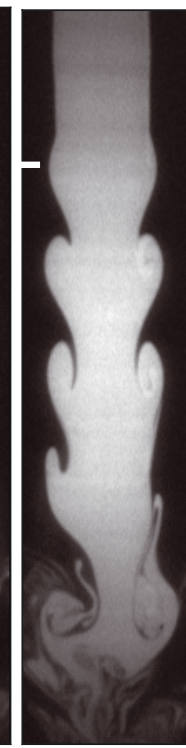

-2 -

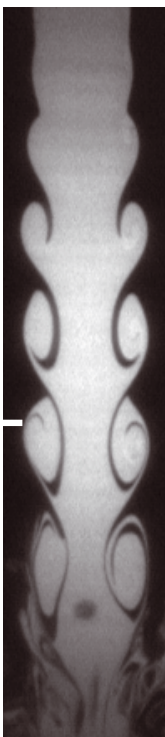

$-8-$

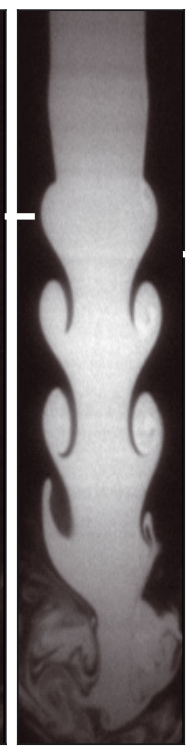

$-3-$

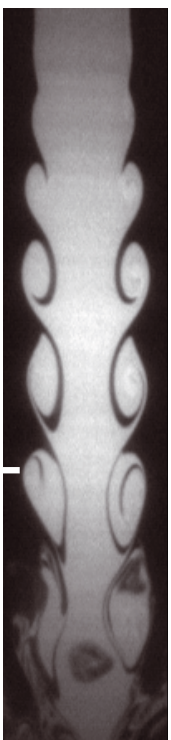

$-9-$

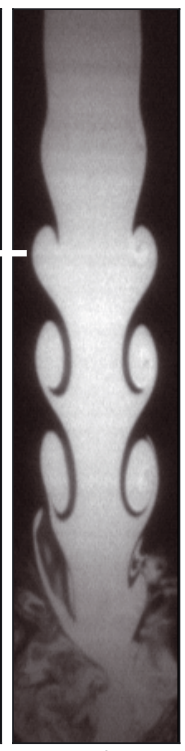

$-4-$

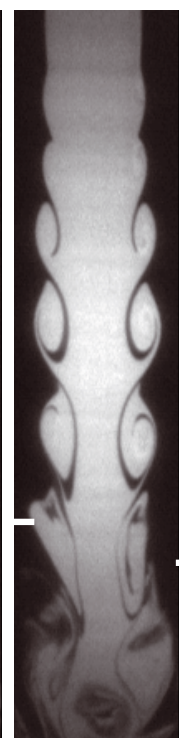

$-10-$

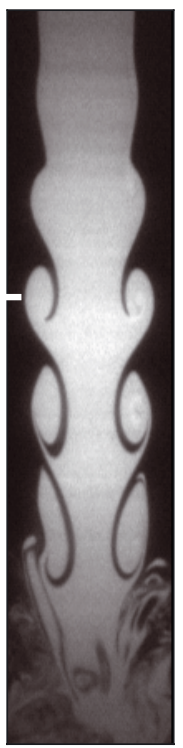

$-5-$

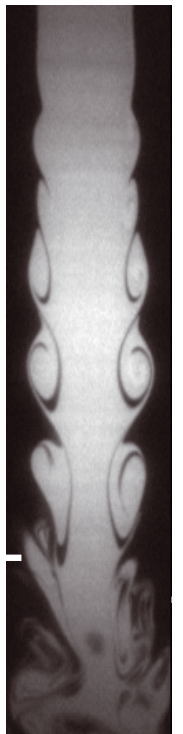

$-11-$
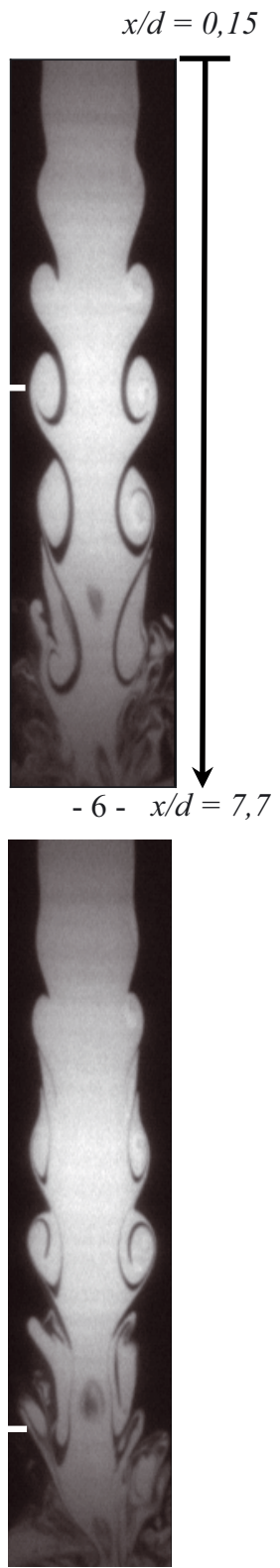

$-12-$

Fig. 3. Évolution temporelle des instabilités de Kelvin-Helmholtz ( $R e=1700, \Delta t=4,54 \mathrm{~ms})$.

\subsubsection{Détection des frontières du jet et localisation des points particuliers}

La frontière du jet est extraite par segmentation des images. Nous avons sélectionné puis mis en concurrence trois méthodes de segmentation pour exploiter les images. La méthode 1 est basée sur un seuillage adaptatif permettant une binarisation efficace des images lorsque le contraste n'est pas uniforme (Marr [17], Zaouali [18]). La méthode 2 utilise un algorithme de segmentation par ondelettes qui permet d'extraire les contours d'une image (Ducottet [19]). La méthode 3 consiste à appliquer un opérateur de palpage morphologique sur les images (Barat [20], Barat [21]). Un exemple de résultats de détection de contour est présenté sur la figure 7 pour un jet évoluant à un nombre de Reynolds $R e=1600$.

La méthode 2 montre une capacité de bien segmenter la totalité des contours. La méthode 1 présente l'avantage d'être simple et la méthode 3 apporte un profil moins bruité.

Les images segmentées sont ensuite analysées pour détecter ses points caractéristiques. À partir des profils obtenus, il est possible, par un simple traitement, de localiser les points caractéristiques des instabilités. La technique consiste à déterminer les points de retournement (les sommets des courbures sur les contours du jet correspondent à des dérivées nulles) (Fig. 8).

L'étude menée ici a porté sur trois séries de 100 images chacune (temps inter-image de 4,54 ms). Sur les trois 


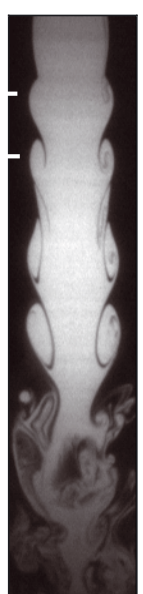

$-1-$

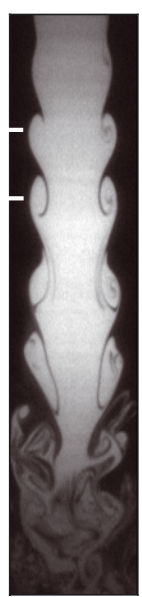

-2 -

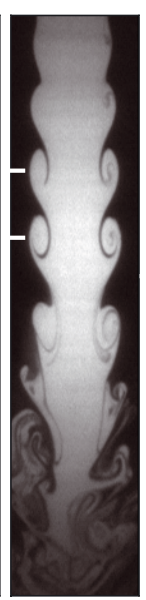

$-3-$

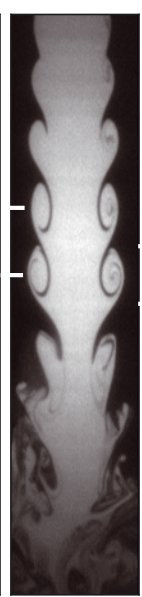

$-4-$

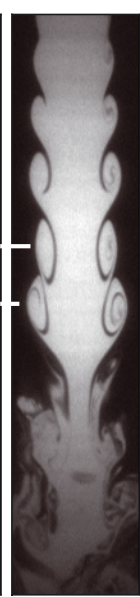

$-5-$

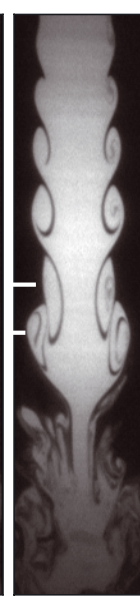

$-6-$

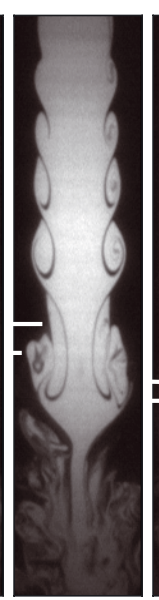

$-7-$

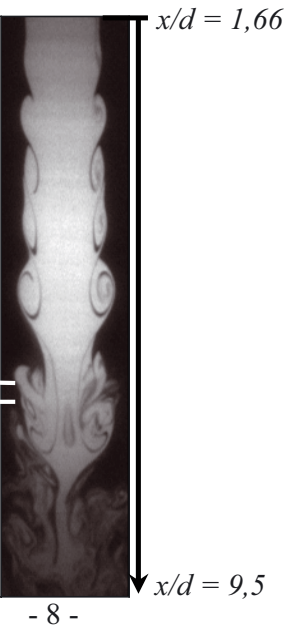

Fig. 4. Suivi temporel de deux motifs ( $R e=1600, \Delta t=4,54 \mathrm{~ms})$.

séries, pour chaque profil (côté droit et côté gauche de part et d'autre de l'axe du jet), les coordonnées en pixels de chaque point $\mathrm{B}_{1}$ ont été enregistrées. Afin de connaître le taux de détection, une valeur moyenne du nombre de points détectés par profil a été calculée, ainsi que l'écart type de ces valeurs (Tab. 2).

Nous pouvons remarquer la bonne concordance des résultats entre les profils côté droit et côté gauche du jet et entre les séries malgré une valeur des écarts types importante.

\subsubsection{Analyse des sommets des crêtes (points $\mathrm{B}_{1}, \mathrm{~B}_{1}^{\prime}, \mathrm{B}_{1}^{\prime \prime}, \ldots$ )}

Sur chacune des 300 images, le traitement de segmentation a été appliqué. Sur les profils obtenus, la localisation des points $\mathrm{B}_{1}$ a été réalisée.

Pour chaque série, l'ensemble des points $\mathrm{B}_{1} \mathrm{du}$ profil côté droit de l'axe du jet de toutes les images a été regroupé sur une même figure, idem pour le profil côté gauche. La figure 9a montre les regroupements des images de la série 1, la figure 9b, ceux des images de la série 2 et la figure 9c, ceux des images de la série 3. L'ordonnée de ces figures indique la position des points dans un axe perpendiculaire à l'axe du jet (axe $Y$ ). En abscisse, l'axe $X$ est colinéaire à l'axe du jet.

Ces regroupements de points $B_{1}$ vont permettre de mesurer un paramètre important de ces instabilités de Kelvin-Helmholtz : l'angle d'amplification. Ce paramètre est noté par l'angle d'ouverture des crêtes de ces instabilités par rapport à la taille du jet à la sortie de la buse (Fig. 10). Pour cela, nous avons appliqué une régression linéaire sur les nuages de points pour obtenir une droite caractérisant l'évolution générale de ces points. Ainsi, l'angle d'amplification $\alpha$ peut être déduit.

Sur ces 6 regroupements, l'angle d'amplification $\alpha$ peut être mesuré. Les résultats sont présentés sur le tableau 3.
Il existe une bonne concordance entre les mesures sur les trois séries. Toutefois, la différence des angles entre les profils côté droit et les profils côté gauche est importante. Ceci est certainement dû au fait que le jet est légèrement orienté en raison de quelques imperfections expérimentales. Mais nous pensons que ces imperfections ne mettent pas en cause la qualité des mesures réalisées. Par conséquent, seule la moyenne de ces valeurs est à conserver.

L'étude des évolutions spatio-temporelles des instabilités de Kelvin-Helmholtz dans un jet d'air libre rond à faible nombre de Reynolds a été abordée dans ce travail au moyen de deux principaux outils : la tomographie laser et les traitements d'images. La structure du jet ainsi que les deux modes d'instabilité sinueux et variqueux ont été étudiés. L'évolution spatio-temporelle des structures et des instabilités de Kelvin-Helmholtz a été suivie en détails. Des phases du scénario de grossissement et de fusion des structures ont été mises en évidence comme dernière étape de la transition à la turbulence.

\section{Conclusion}

D'après ces visualisations, il nous semble que chaque structure ou « motif » qui apparaît sur la frontière du jet, a sa propre «vie». Par ailleurs, la majorité des motifs prend naissance sur les bords du jet et subit presque le même avenir.

Nous avons pu également faire des mesures de quelques paramètres caractéristiques des instabilités à partir des images acquises malgré leur résolution moyenne $(640 \times 100$ pixels $)$.

À ce stade, seuls les points $\mathrm{B}_{1}$, les crêtes, ont été localisés. Bien entendu, il apparaît nécessaire de faire le même travail sur les autres points caractéristiques des instabilités : les points $\mathrm{C}$, les points $\mathrm{P}, \ldots$ (Creux, Pointes, ...), et ce par amélioration des algorithmes utilisés dans nos traitements et qui fournissent des résultats moyens dans 

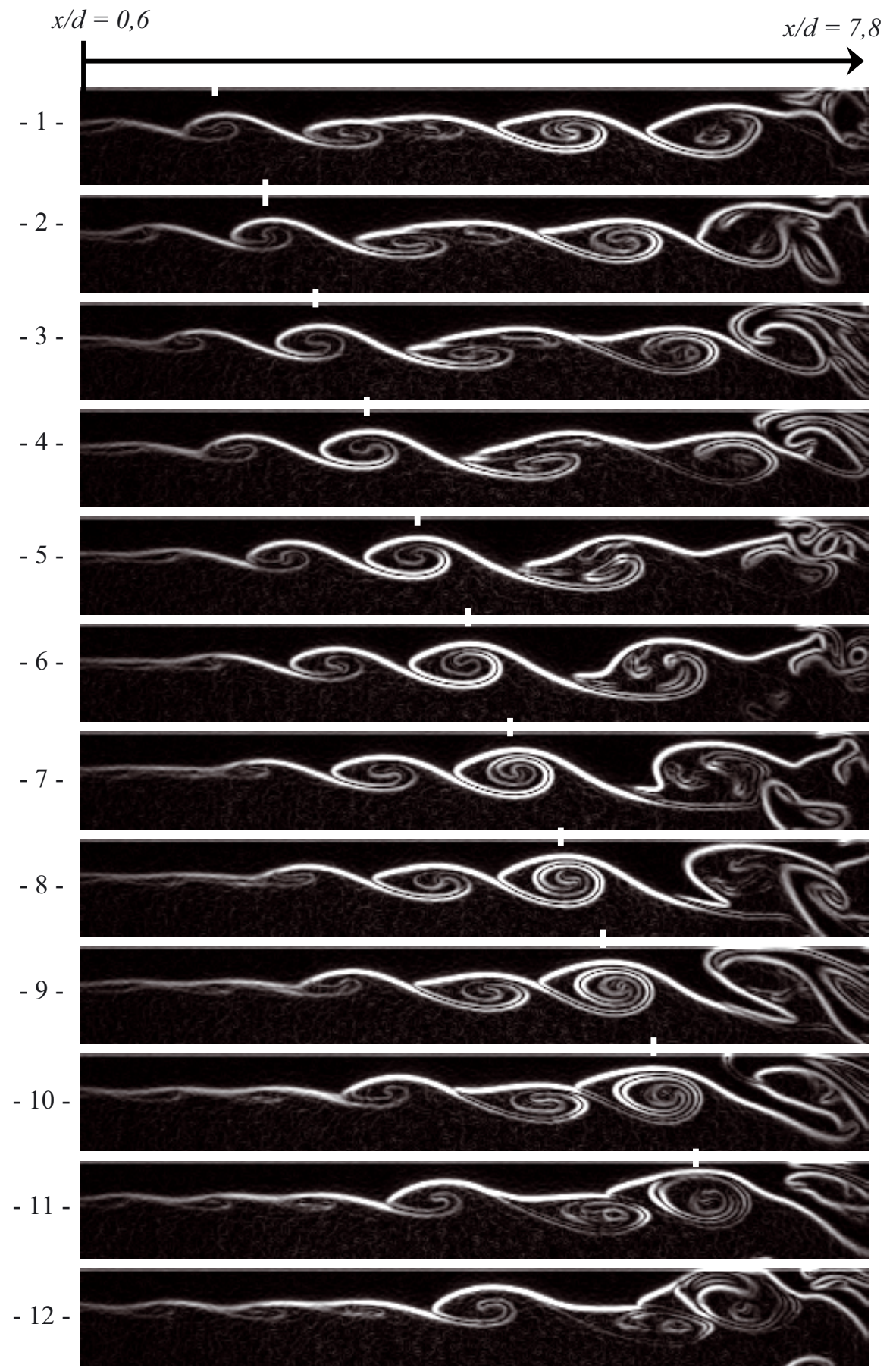

Fig. 5. Évolution temporelle des structures tourbillonnaires ( $R e=1300, \Delta t=4,54 \mathrm{~ms})$.

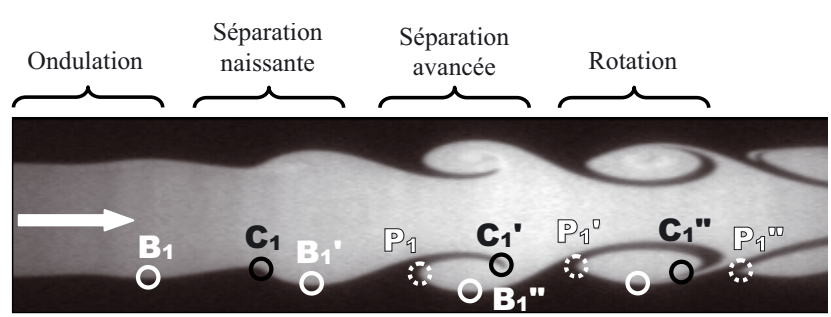

B : Bosse ; C : Creux ; P : Pointe

Fig. 6. Description des différents points caractéristiques et formes des instabilités. les zones de « séparation avancée » et de « rotation ». Ces informations permettraient de quantifier de manière plus complète l'évolution spatio-temporelle de ces instabilités.

L'ensemble de ces données apportera, on l'espère, des moyens de mieux appréhender les mécanismes qui donnent naissance à ces instabilités.

Remerciements. Que Madame Cécile Barat, Messieurs Christophe Ducottet et Michel Courbon du Laboratoire Hubert Curien de Saint-Étienne, reçoivent nos remerciements 
Tableau 1. Paramètres caractéristiques des instabilités.

\begin{tabular}{|c|c|c|c|}
\hline & $R e=1500$ & $R e=1600$ & $R e=1700$ \\
\hline $\begin{array}{l}\text { Déplacement moyen } \Delta x \\
\text { (en mm à } 0,5 \text { mm près) }\end{array}$ & 5,54 & 5,74 & 6,41 \\
\hline$\Delta x / d$ & 0,44 & 0,46 & 0,51 \\
\hline Vitesse moyenne $U\left(\mathrm{~m} \cdot \mathrm{s}^{-1}\right)$ & 1,22 & 1,26 & 1,41 \\
\hline$U / U_{0}$ & 0,65 & 0,63 & 0,66 \\
\hline $\begin{array}{l}\text { Longueur d'onde moyenne } \lambda \\
\text { (en mm à } 0,5 \text { mm près) }\end{array}$ & 12,46 & 12,96 & 12,54 \\
\hline$\lambda / d$ & 1,00 & 1,05 & 1,01 \\
\hline
\end{tabular}

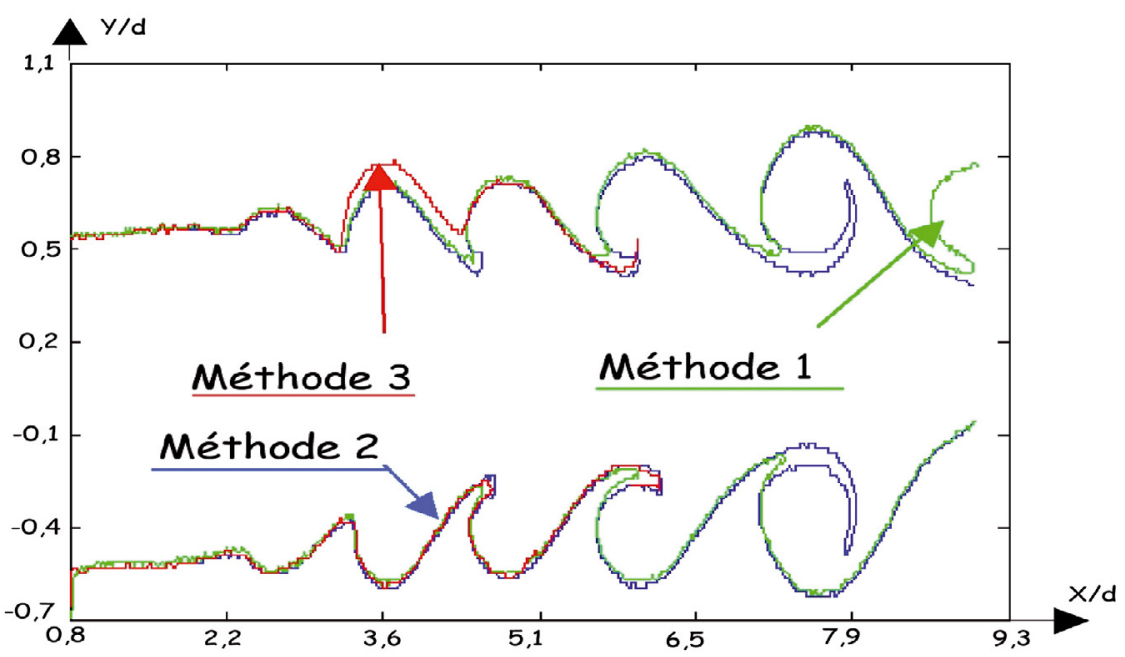

Fig. 7. Extraction des contours du jet par segmentation $(R e=1600)$.

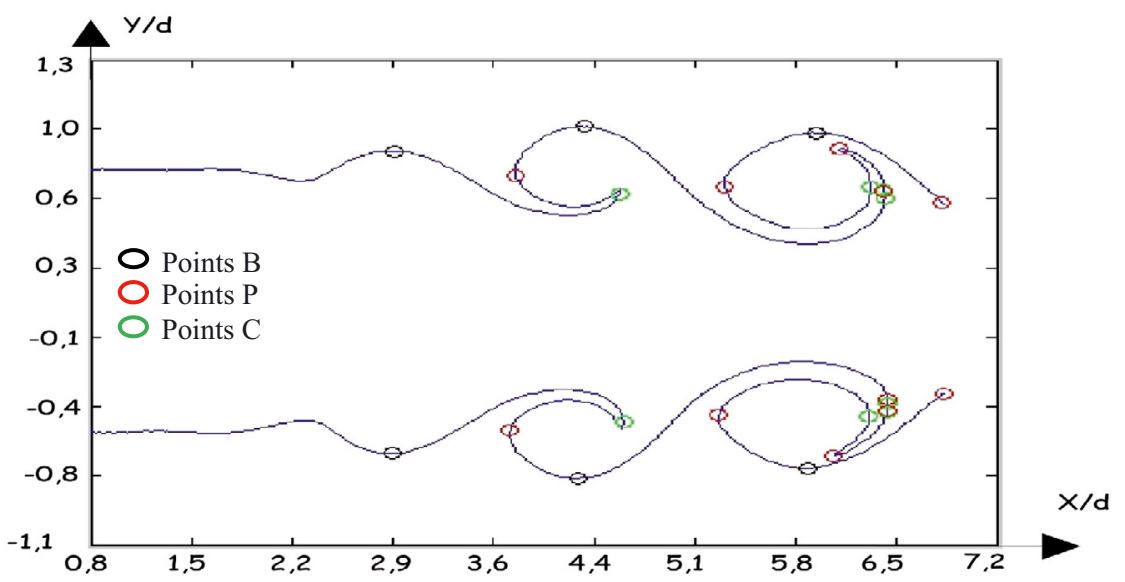

Fig. 8. Contours du jet et ses points caractéristiques $(R e=1600)$ (la segmentation du profil a été obtenue par la méthode 1).

Tableau 2. Nombre de points détectés par profil.

\begin{tabular}{cccc}
\hline Série & Profil & $\begin{array}{c}\text { Moyenne du nombre } \\
\text { de points détectés }\end{array}$ & Écart type \\
\hline 1 & «droit & 3,87 & 1,57 \\
1 & « gauche & 3,99 & 1,59 \\
2 & «droit » & 3,17 & 1,87 \\
2 & «gauche» & 3,26 & 2,10 \\
3 & «droit » & 4,73 & 1,09 \\
3 & «gauche & 4,71 & 1,42 \\
\hline
\end{tabular}


Tableau 3. Angle d'amplification $\alpha$.

\begin{tabular}{|c|c|c|c|}
\hline Série & Profil & Angle d'amplification $\alpha\left(\mathrm{en}^{\circ}\right)$ & Moyenne $\left(\mathrm{en}^{\circ}\right)$ \\
\hline 1 & 《droit » & $2,22^{\circ}$ & $0,98^{\circ}$ \\
\hline 1 & «gauche » & $-0,26^{\circ}$ & \\
\hline 2 & 《droit $»$ & $1,8^{\circ}$ & $1,20^{\circ}$ \\
\hline 2 & «gauche » & $0,6^{\circ}$ & \\
\hline 3 & 《droit » & $2,36^{\circ}$ & $1,26^{\circ}$ \\
\hline 3 & «gauche » & $0,16^{\circ}$ & \\
\hline
\end{tabular}
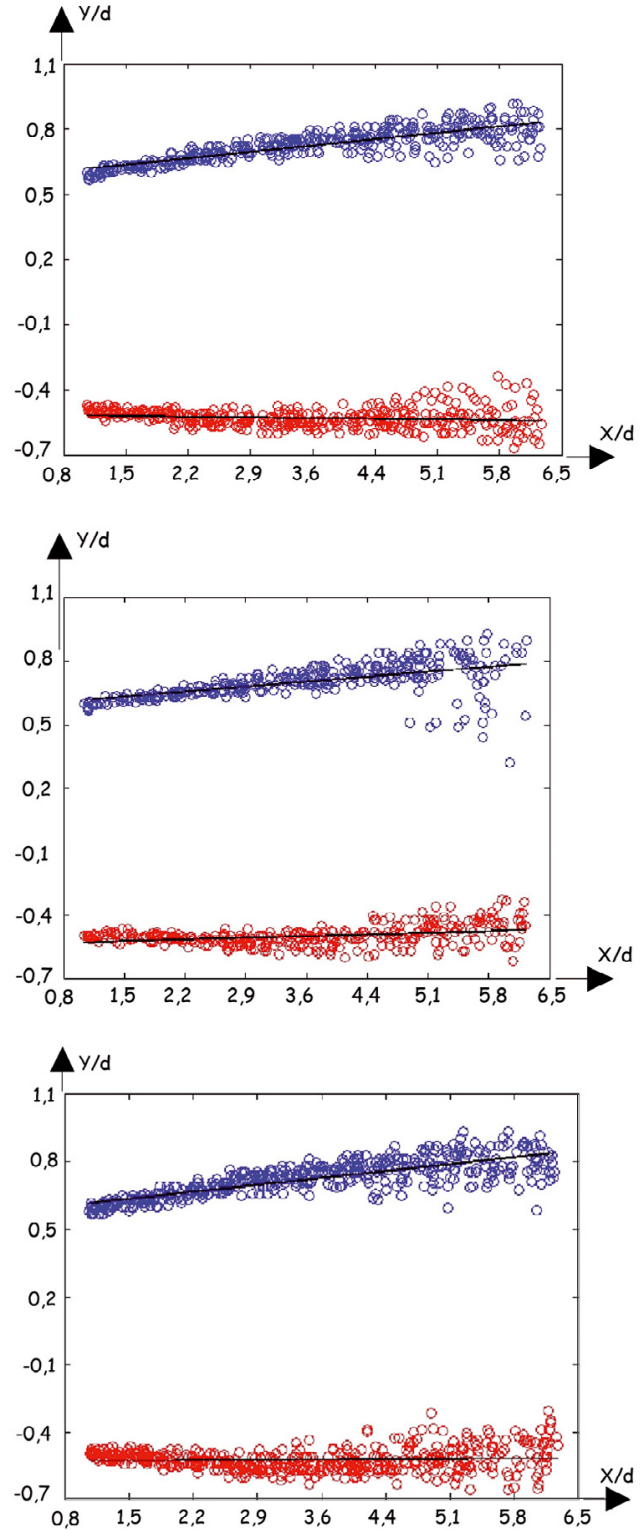

Fig. 9. Regroupements des points $B_{1}$. (a) Série 1. (b) Série 2. (c) Série 3.

les plus chaleureux pour leur aide précieuse notamment dans le domaine de traitement d'images. Nos remerciements sont adressés également à Monsieur le Professeur Jean-Paul Schon pour l'intérêt constant qu'il a porté à notre travail.

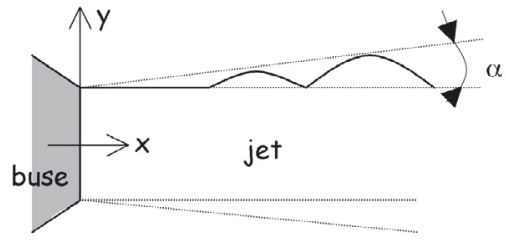

Fig. 10. Définition de l'angle d'amplification $\alpha$.

\section{Références}

[1] G.K. Batchelor, A.E. Gill, Analysis of the stability of axisymmetric jets, J. Fluid Mech. 14 (1962) 529-551

[2] S.C. Crow, F.H. Champagne, Orderly structure in jet turbulence, J. Fluid Mech. 48 (1971) 547-591

[3] J. Cohen, I. Wygnanski, The evolution of instabilities in the axisymmetrical jet Part I. The linear growth of disturbances near the nozzle, J. Fluid Mech. 176 (1987) 191-219

[4] J.C. Mollendorf, B. Gebhart, An experimental and numerical study of the viscous stability of a round laminar vertical jet with and without thermal buoyancy for symmetric and asymmetric disturbances, J. Fluid Mech. 61 (1973) 367-399

[5] H. Ben Aissia, Y. Zaouali, J. Jay, T. Fournel, P. Gervais, Étude expérimentale par visualisation et Anémométrie Laser d'un jet axisymétrique, Les Annales Maghrébines de l'Ingénieur 14 (2000) 29-38

[6] J. Fontaine, Transition des écoulements cisaillés libres à densité variable, Thèse de Doctorat, Institut National Polytechnique de Toulouse, 2005

[7] I. Danaila, Étude des instabilités et des structures cohérentes dans la zone de proche sortie d'un jet axisymétrique, Thèse de doctorat, Université de la Méditerranée Aix-Marseille II, France, 1997

[8] J.O. Hinze, Turbulence, 2nd Edition, McGraw-Hill, New York, 1975

[9] H. Ben Aissia, Y. Zaouali, S. El Golli, Numerical study of the influence of dynamic and thermal exit conditions on axisymmetric laminar buoyant jet, Numerical Heat Transfer: Applications 42 (2002) 427-444

[10] S. Ravier, Étude des instabilités dans un jet plan à masse volumique variable, Thèse de Doctorat, Université AixMarseille II, France, 2004

[11] H.A. Becker, T.A. Massaro, Vortex evolution in a round jet, J. Fluid Mech. 31 (1968) 435-448

[12] G.E. Mattingly, C.C. Chang, Unstable waves on an axisymmetric jet column, J. Fluid Mech. 65 (1974) 541-560

[13] A.J. Reynolds, Observation of a liquid-into-liquid jet, J. Fluid Mech. 14 (1962) 552-561

[14] H. Ben Aissia, Étude numérique et expérimentale par imagerie et Anémométrie Laser Doppler d'un jet axi- 
symétrique, Thèse de Doctorat d'État, Université Tunis El-Manar, Faculté des Sciences de Tunis, Tunisie, 2002

[15] Y. Zaouali, H. Ben Aissia, N. Kechiche, J. Jay, J.-P. Schon, Experimental study of the instabilities in the laminar turbulent transition zone of an axisymmetric jet at low Reynolds number, J. Flow Visualization and Image Processing 11 (2004) 1-16

[16] C-M. Ho, P. Huerre, Perturbed free shear layers, Ann. Rev. Fluid Mech. 16 (1984) 365-422

[17] D. Marr, E. Hildreth, Theory of edge detection, Proceeding of the Royal Society, London, B207, 1980, pp. $187-217$

[18] Y. Zaouali, N. Filali, H. Ben Aissia, J. Jay, M. Courbon, C. Barat, C. Ducottet, Caractérisation des évolutions spatio-temporelles des instabilités de Kelvin-Helmholtz dans un jet rond, $11^{\mathrm{e}}$ colloque de Visualisation et de Traitement d'Images en Mécanique des Fluides : FluVisu11, Écully, France, 2005

[19] C. Ducottet, T. Fournel, C. Barat, Scale-adaptive detection and local characterization of edges based on wavelet transform, Signal Processing 84 (2004) 2115-2137

[20] C. Barat, C. Ducottet, M. Jourlin, Mise en correspondance de formes à niveaux de gris par palpage morphologique, GRETSI, Paris, 2003, pp. 138-142

[21] C. Barat, C. Ducottet, M. Jourlin, Line Pattern Segmentation Using Morphological Probing, Third International Symposium on Image and Signal Processing and Analysis, Rome, Italie, 2003, pp. 417-422 